\title{
A NONSTANDARD CHARACTERIZATION OF WEAK CONVERGENCE
}

\author{
ROBERT M. ANDERSON AND SALIM RASHID ${ }^{1}$
}

\begin{abstract}
Let $X$ be any topological space, and $C(X)$ the space of bounded continuous functions on $X$. We give a nonstandard characterization of weak convergence of a net of bounded linear functionals on $C(X)$ to a tight Baire measure on $X$. This characterization applies whether or not the net or the individual functionals in the net are tight. Moreover, the characterization is expressed in terms of the values of an associated net of countably additive measures on all Baire sets of $X$; no distinguished family, such as the family of continuity sets of the limit, is involved. As a corollary, we obtain a new proof that a tight set of measures is relatively weakly compact.
\end{abstract}

1. Introduction. Let $X$ be an arbitrary topological space, $C(X)$ the Banach space of all bounded continuous real-valued functions on $X$ (with the sup norm), and $C(X)^{d}$ the space of bounded linear functionals on $C(X)$. The weak topology (or more precisely, the weak-star topology) on $C(X)^{d}$ is the topology generated by the subbase $\{\{\phi: \phi(f)<\alpha\}, f \in C(X), \alpha \in \mathbf{R}\}$. Thus, a net $\left\{\phi_{\alpha}\right\}_{\alpha \in D}$ converges weakly to $\phi$ (written $\phi_{\alpha} \Rightarrow \phi$ ) if and only if $\phi_{\alpha}(f) \rightarrow \phi(f)$ for all $f \in C(X)$. This notion is of fundamental importance in probability, where one considers the weak topology relativized to the space of all Baire (or Borel) probability measures on $X$. If $\left\{\mu_{\alpha}\right\}$ is a net of measures, $\mu_{\alpha} \Rightarrow \mu$ if and only if $\int f d \mu_{\alpha} \rightarrow \int f d \mu$ for all $f \in C(X)$.

In this paper, we give a nonstandard characterization of weak convergence of a net $\left\{\phi_{\alpha}\right\}_{\alpha \in D}$ in $C(X)^{d}$ to a tight countably additive Baire measure. The characterization involves an associated net $\left\{\mu_{\alpha}\right\}_{\alpha \in *^{*}}$ of countably additive Baire measures on $X . \mu_{\alpha}$ is obtained from a Loeb measure as introduced by Peter A. Loeb [5] and a measure-preserving map. Measure-preserving maps in nonstandard analysis were first used in special cases by Loeb [6] followed by the first author [1]; a general theory based on them will be given in [2].

One aspect of the characterization is particularly noteworthy. Even in the

Received by the editors May 18, 1977 and, in revised form, September 21, 1977.

AMS (MOS) subject classifications (1970). Primary 02H25, 26A98, 60B05, 60B10.

Key words and phrases. Weak convergence, tight, relatively weakly compact, topological measure theory, nonstandard analysis.

${ }^{1}$ Portions of this material were developed independently by the authors and included in dissertations submitted for the degree of Doctor of Philosophy in Yale University. The authors are grateful to Professors Yoav Benyamini, Donald J. Brown, S. Kakutani and Ward Whitt for a number of very profitable discussions, and to the referee for a number of suggestions which improved the exposition. The first author gratefully acknowledges the support of a Canada Council Doctoral Fellowship. 
simplest cases, standard characterizations involve a subclass of the Baire sets which depends on the limit measure. For example, if $\left\{P_{n}\right\}$ is a sequence of probability measures on $\mathbf{R}, P_{n} \rightarrow P$ does not imply $P_{n}(A) \rightarrow P(A)$ for all Baire or even all open Baire $A$. Indeed, we can guarantee $P_{n}(A) \rightarrow P(A)$ only when $A$ is a continuity set for $P$, i.e. the topological boundary of $A$ is a $P$-null set. Note that the class of continuity sets is not defined intrinsically in terms of the sequence $\left\{P_{n}\right\}$, but only in terms of the limit $P$.

In the nonstandard characterization, all Baire sets are treated alike. We shall see that $\phi_{\alpha}$ converges weakly essentially if $\mu_{\alpha}(B)=\mu_{\beta}(B)$ for all infinite $\alpha, \beta \in{ }^{*} D$ and all Baire $B \subset X$ (an element $\alpha \in{ }^{*} D$ is said to be infinite if $\alpha \geqslant \beta$ for all $\beta \in D$ ).

Based on the characterization, we give a new proof of a result of Varadarajan, that a tight set of functionals is relatively weakly compact in the space of Baire measures.

Special cases of these results have been treated independently by the authors. In his dissertation [9], the second author gave a similar characterization of weak convergence in compact metric spaces. In [1], the first author used the essential elements of the proof in giving a new proof of Donsker's Theorem on the weak convergence of random walks to Brownian motion. The general results in this paper appear in the dissertation of the first author [3].

Loeb has pointed out that a result related to Lemma 2(iii) appeared in a paper of his concerning potential theory [6, Theorem 4.9]. He showed that maximal representing measure, obtained in a fashion analogous to our construction of $\mu_{\phi}$ was tight and gave approximately correct integrals to certain special continuous functions. The technique he used to calculate these integrals is the same as ours. This part of Loeb's work was completed prior to our work on weak convergence.

D. W. Müller [8] has given a nonstandard characterization of tight measures and used this to give a nonstandard proof of the Donsker-Prokhorov invariance principle. Müller's condition for tightness forms an important part of the characterization given here. Our result extends his in the following ways: it gives a characterization of weak convergence itself, rather than tightness; it applies to nontight nets of measures; and it applies to topological spaces, instead of metric spaces.

We shall assume that the reader is familiar with nonstandard analysis. An excellent introduction to the subject is given by Stroyan and Luxemburg [10].

2. Characterization of weak convergence. Let $\left(X, \mathscr{T}^{\prime}\right)$ be an arbitrary topological space. The Baire algebra $\mathscr{B}$ on $X$ is the smallest $\sigma$-algebra such that every $f \in C(X)$ is $\mathscr{B}$-measurable. We may replace $\mathcal{T}^{\prime}$ by $\mathcal{T}$, the weakest topology on $X$ such that every $f \in C(X)$ is continuous. Thus, $\mathscr{T}$ is generated by the subbase $\{\{x \in X: f(x)<\alpha\}, f \in C(X), \alpha \in \mathbf{R}\}$. This does not change $B, C(X), C(X)^{d}$, or the weak topology on $C(X)^{d}$. Any topological terms used in what follows will be understood with respect to $\mathcal{T}$ rather than 
$\mathcal{T}^{\prime}$. Note that, since $\mathcal{T} \subset \mathcal{T}^{\prime}$, any $\mathcal{T}^{\prime}$-compact set is compact.

Let $\mathcal{F}$ be the algebra generated by $\{\{x \in X: f(x)<\alpha\}, f \in C(X), \alpha \in$ $\mathbf{R}$ \}. It is well known [11, Theorem 6, p. 165] that, if $\phi \in C(X)^{d}$, there is a finitely additive measure $\nu_{\phi}: \mathcal{F} \rightarrow \mathbf{R}$ such that $\phi(f)=\int_{X} f d \nu_{\phi}$ for all $f \in$ $C(X)$.

If $\mu$ is any measure, let $\|\mu\|$ denote the total variation norm. Let $\mathfrak{N}$ be the set of countably additive Baire measures such that $\|\mu\|$ is finite. We may consider $\Re$ as a subset of $C(X)^{d}$.

Let ${ }^{*} X$ be the nonstandard extension of $X$ in a $\kappa$-saturated enlargement, where $\mathcal{T}$ has a base of cardinality $<\kappa$. For any $x \in X$, let $m(x)$ denote the monad of $x, \cap_{T \in \mathcal{T} ; x \in T}{ }^{*} T$. The standard part relation is defined by $x=$ st $(y)$ if $y \in m(x)$. Since $X$ need not be Hausdorff, st need not be a function. However, if $B, C \in \mathscr{B}$, and $B \cap C$ is empty, then $\operatorname{st}^{-1}(B) \cap \mathrm{st}^{-1}(C)$ is empty.

Suppose $\nu_{\phi}$ is an internal finitely additive measure on $* \mathcal{F}$ and ${ }^{\circ}\left\|\nu_{\phi}\right\|<\infty$. Let $L\left(\nu_{\phi}\right)$ denote the Loeb measure generated by $\nu_{\phi}$, i.e. the completion of the Caratheodory extension of ${ }^{\circ} \nu_{\phi}$ to a countably additive measure on $\sigma(* \mathscr{F})$, the $\sigma$-algebra generated by $* \mathscr{F}$; let $L(* \mathscr{F})$ be the algebra of measurable sets in this completion. For details of the construction of Loeb measures, see [5].

Suppose $T \subset X$. Write $f \subset T$ if $f \in C(X),\|f\| \leqslant 1$ and $f(x)=0$ for all $x \notin T . A \subset C(X)^{d}$ is said to be tight if $\{\|\phi\|: \phi \in A\}$ is bounded and, for each $\varepsilon \in \mathbf{R}_{+}$, there exists a compact $K \subset X$ such that $|\phi(f)|<\varepsilon$ for all $\phi \in A$ and all $f \subset K^{c} . \phi \in C(X)^{d}$ is said to be tight if $\{\phi\}$ is. Let $\mathfrak{T}_{t}=\{\mu$ $\in M: \mu$ is tight $\}. \phi \in{ }^{*} C(X)^{d}$ is said to be $S$-tight if ${ }^{\circ}\|\phi\|<\infty$ and, for each $\varepsilon \in R_{+}$, there exists $K \subset X$ compact such that $f \subset K^{c}$ implies $\left|\phi\left({ }^{*} f\right)\right|<$ $\varepsilon$. An internal finitely additive measure $\nu_{\phi}$ on $* \mathscr{F}$ is said to be near-standardly concentrated if $\left|L\left(\nu_{\phi}\right)\right|\left({ }^{*} X-\mathrm{ns}\left({ }^{*} X\right)\right)=0$.

Suppose $\nu_{\phi}$ is near-standardly concentrated. If $f \in C(X)$,

$$
\begin{aligned}
\mathrm{st}^{-1}(\{x \in X: f(x)<\alpha\}) & =\left\{x \in \mathrm{ns}\left({ }^{*} X\right):{ }^{\circ}{ }^{*} f(x)<\alpha\right\} \\
=\mathrm{ns}\left({ }^{*} X\right) & \cap\left(\bigcup_{n=1}^{\infty}\left\{x \in{ }^{*} X:{ }^{*} f(x)<\alpha-\frac{1}{n}\right\}\right) \in L\left({ }^{*} \mathscr{F}\right) .
\end{aligned}
$$

Hence, for any $B \in \mathscr{B}, \mathrm{st}^{-1}(B) \in L(* \mathscr{F})$. Thus, $\mu_{\phi}(B)=L\left(\nu_{\phi}\right)\left(\mathrm{st}^{-1}(B)\right)$ defines a countably additive measure on $\mathscr{B}$; in other words, $\mu_{\phi} \in \mathfrak{N}$.

The proof of the following lemma is elementary and will be omitted.

LEMMA 1. If $x \in T \in \mathcal{T}$, there exists a neighbourhood $V$ of $x$ and $f \in$ $C(X), 0 \leqslant f \leqslant 1$, such that $\left.f\right|_{V}=0$ and $\left.f\right|_{T^{c}}=1$. In particular, $X$ is regular.

Lemma 2 (Compare Müller [8, Lemma 2].) (i) Suppose $A$ is a tight subset of $C(X)^{d}$. Then any $\phi \in{ }^{*} A$ is $S$-tight.

(ii) If $\phi \in{ }^{*} C(X)^{d}$ is $S$-tight, then $\nu_{\phi}$ is near-standardly concentrated.

(iii) If $\nu_{\phi}$ is near-standardly concentrated, then $\mu_{\phi} \in \mathfrak{R}_{t}$ and $\phi\left({ }^{*} f\right) \simeq$ 
$\int_{x} f d \mu_{\phi}$ for all $f \in C(X)$; in other words, $\phi$ is in the weak-star monad of $\mu_{\phi}{ }^{2}$

Proof. It is well known [11, Theorems 4, 7, p. 165] that $\phi$ may be decomposed as the difference of positive linear functionals, and that $\phi$ is tight ( $S$-tight) if and only if both terms of the decomposition are tight ( $S$-tight). Hence, we may assume that $\phi$ is a positive linear functional. Pick a standard $\varepsilon>0$.

(i) is trivial.

(ii) There exists $K$ compact such that $f \subset K^{c}$ implies $\phi\left({ }^{*} f\right)<\varepsilon$. For $K \subset T \in \mathcal{T}$, let $\mathscr{F}_{T}=\left\{S \in{ }^{*} \mathscr{F}:{ }^{*} K \subset S \subset{ }^{*} T, \nu_{\phi}\left(S^{c}\right)<\varepsilon\right\}$. We shall show that $\mathscr{F}_{T}$ is nonempty.

If $k \in K$, there exists a neighbourhood $V_{k}$ of $k$ and $f_{k} \in C(X)$ with $0<f_{k}<1,\left.f_{k}\right|_{V_{k}}=0$ and $\left.f_{k}\right|_{T^{c}}=1$, by Lemma 1 . Since $K$ is compact, a finite collection $V_{k_{1}}, \ldots, V_{k_{n}}$ covers $K$. Let $f=f_{k_{1}} \wedge \cdots \wedge f_{k_{n}}$. Then $f \subset K^{c}$ and $\left.f\right|_{T^{c}}=1$. Let $S=\left\{x \in{ }^{*} X:{ }^{*} f(x)<1\right\}$. Then $S \in{ }^{*} \mathscr{F},{ }^{*} K \subset S$ $\subset^{*} T$.

$$
\nu_{\phi}\left(S^{c}\right) \leqslant \int_{*_{X}}^{*} f d \nu_{\phi}=\phi\left({ }^{*} f\right)<\varepsilon
$$

Hence $S \in \mathscr{F}_{T}$.

If $K \subset T_{i} \in T(1 \leqslant i \leqslant n)$, then $\mathscr{F}_{T_{1}} \cap \cdots \cap \mathscr{F}_{T_{n}}=\mathscr{F}_{T_{1} \cap \cdots \cap T_{n}}$ is nonempty. Since $\mathcal{T}$ has a base of cardinality $<\kappa$ and ${ }^{*} X$ is $\kappa$-saturated, $\cap_{K \subset T \in \sigma^{F}} \mathscr{F}_{T}$ is nonempty. Thus, there exists

$$
S \in{ }^{*} \mathscr{F}, \quad \nu_{\phi}\left(S^{c}\right)<\varepsilon, \quad{ }^{*} K \subset S \subset \bigcap_{K \subset T \in \mathscr{T}}{ }^{*} T=\bigcup_{k \in K} m(k)
$$

by [7, Theorem 3.5.2]. Thus, $S \subset \operatorname{ns}\left({ }^{*} X\right)$. Since $\varepsilon$ is arbitrary and $L\left(\nu_{\phi}\right)$ is complete, $\nu_{\phi}$ is near-standardly concentrated.

(iii) By [5, Theorem 1], there exists $A \in{ }^{*} \mathscr{F}$ such that $A \subset \mathrm{ns}\left({ }^{*} X\right),{ }^{\circ} \nu_{\phi}\left(A^{c}\right)$ $<\varepsilon$. Let $K=\operatorname{st}(A)$. By [7, Theorem 3.7.1], $K$ is compact. If $f \subset K^{c}$ and $x \in A,{ }^{*} f(x) \simeq f\left({ }^{\circ} x\right)=0$. Therefore

$$
\begin{aligned}
\int_{X} f d \mu_{\phi} & =\int_{\mathrm{ns}\left({ }^{*} X\right)} f\left({ }^{\circ} x\right) d L\left(\nu_{\phi}\right)=\int_{\mathrm{ns}\left({ }^{*} X\right)}{ }^{\circ *} f(x) d L\left(\nu_{\phi}\right) \\
& =\int_{{ }^{*} X}{ }^{\circ *} f(x) d L\left(\nu_{\phi}\right) \leqslant L\left(\nu_{\phi}\right)\left(A^{c}\right)={ }^{\circ} \nu_{\phi}\left(A^{c}\right)<\varepsilon .
\end{aligned}
$$

Hence $\mu_{\phi} \in \Re_{t}$.

For any $f \in C(X)$,

$$
\begin{aligned}
\phi\left({ }^{*} f\right) & =\int_{{ }^{*} X}{ }^{*} f d v_{\phi} \simeq \int_{{ }^{*} X}{ }^{\circ *} f d L\left(\nu_{\phi}\right) \quad(\text { by }[5, \text { Theorem 3] }) \\
& =\int_{\mathrm{ns}\left({ }^{*} X\right)}{ }^{\circ *^{*}} f d L\left(\nu_{\phi}\right)=\int_{\mathrm{ns}\left({ }^{*} X\right)} f\left({ }^{\circ} x\right) d L\left(\nu_{\phi}\right)=\int_{X} f d \mu_{\phi} .
\end{aligned}
$$

\footnotetext{
${ }^{2}$ Note added in proof. This statement of the result in terms of the weak-star monad was suggested by Loeb. This point of view is central to his forthcoming article, "Weak limits and the standard part map".
} 
Definition 3. Let $\left\{\phi_{\alpha}\right\}_{\alpha \in D}$ be a net in $C(X)^{d}$ with $\left\|\phi_{\alpha}\right\|$ bounded. For $\alpha \in 4 D$, let $\nu_{\alpha}=\nu_{\phi_{\alpha}}$; if $\nu_{\alpha}$ is near-standardly concentrated, let $\mu_{\alpha}=\mu_{\phi_{\alpha}}$.

THEOREM 4. Let $\left\{\phi_{\alpha}\right\}_{\alpha \in D}$ be a net in $C(X)^{d}$ with $\left\|\phi_{\alpha}\right\|$ bounded. Then the following are equivalent:

(i) $\phi_{\alpha}$ converges weakly to an element of $\Re_{t}$.

(ii) For all infinite $\alpha, \beta \in{ }^{*} D, \nu_{\alpha}$ is near-standardly concentrated and $\mu_{\alpha}=$ $\mu_{\beta}$.

In this case, the weak limit is the common value of $\mu_{\alpha}$ for infinite $\alpha$.

Proof. Suppose $\phi_{\alpha} \Rightarrow \mu \in \Re_{t}$. If $\alpha$ is infinite, $\phi_{\alpha}\left({ }^{*} f\right) \simeq \int f d \mu$ for all $f \in C(X)$. Hence $\phi_{\alpha}$ is $S$-tight, since $\mu \in \mathfrak{N}_{t}$. By Lemma 2, $\nu_{\alpha}$ is near-standardly concentrated, so $\mu_{\alpha}$ is defined. If $f \in C(X)$,

$$
\int f d \mu_{\alpha} \simeq \phi_{\alpha}\left({ }^{*} f\right) \simeq \int f d \mu \simeq \phi_{\beta}\left({ }^{*} f\right) \simeq \int f d \mu_{\beta}
$$

Therefore

$$
\int f d \mu_{\alpha}=\int f d \mu=\int f d \mu_{\beta} .
$$

Since this is true for all $f \in C(X), \mu, \mu_{\alpha}$, and $\mu_{\beta}$ all agree on all Baire sets, i.e., $\mu_{\alpha}=\mu_{\beta}=\mu$.

Conversely, suppose $\nu_{\alpha}$ is near-standardly concentrated and $\mu_{\alpha}=\mu_{\beta}$ for all infinite $\alpha, \beta$. Let $\mu$ be the common value of $\mu_{\alpha}$. By Lemma 2(iii), $\mu \in \mathfrak{N}_{t}$ and $\phi_{\alpha}\left({ }^{*} f\right) \simeq \int f d \mu$ for all $f \in C(X)$. Hence $\phi_{\alpha}(f) \rightarrow \int f d \mu$, and so $\phi_{\alpha} \Rightarrow \mu$.

Corollary 5. Suppose $X$ is compact and $\left\{\phi_{\alpha}\right\}_{\alpha \in D}$ is a net in $C(X)^{d}$ with $\left\|\phi_{\alpha}\right\|$ bounded. Then $\phi_{\alpha}$ converges weakly to an element of $\Re$ if and only if $\mu_{\alpha}=\mu_{\beta}$ for all infinite $\alpha, \beta \in{ }^{*} D$. In this case, the common value of $\mu_{\alpha}$ for infinite $\alpha$ is the weak limit.

Proof. Since $X$ is compact, $\Re=\Re_{t}$ and $n s\left({ }^{*} X\right)={ }^{*} X$. Hence, the result follows from Theorem 4 .

Theorem 6 (Varadarajan [11, Corollary III, p. 205]). Suppose $A$ is a tight subset of $C(X)^{d}$. Then $A$ is relatively weakly compact in $\mathfrak{N}_{t}$.

Proof. Suppose $\phi \in{ }^{*} A$. By Lemma 2, $\nu_{\phi}$ is near-standardly concentrated and $\mu_{\phi} \in \mathfrak{N}_{t} ; \phi\left({ }^{*} f\right) \simeq \int f d \mu_{\phi}$ for all $f \in C(X)$. Thus, $\phi$ is weakly nearstandard in $* \mathfrak{K}_{t}$. Since $\mathfrak{K}_{t}$ is Hausdorff in the weak topology, $A$ is relatively weakly compact by $[7$, Theorem 3.6 .2$]$.

\section{REFERENCES}

1. Robert M. Anderson, A nonstandard representation for Brownian motion and Itô integration, Israel J. Math. 25 (1976), 15-46.

2. __ Star-finite representations of measure spaces (to appear). 
3. __ Star-finite probability theory, Ph.D. Dissertation, Yale Univ., May, 1977.

4. Patrick Billingsley, Convergence of probability measures, Wiley, New York, 1968.

5. Peter A. Loeb, Conversion from nonstandard to standard measure spaces and applications in probability theory, Trans. Amer. Math. Soc. 211 (1975), 113-122.

6. Applications of nonstandard analysis to ideal boundaries in potential theory, Israel J. Math. 25 (1976), 154-187.

7. W. A. J. Luxemburg, A general theory of monads, Applications of Model Theory to Algebra, Analysis, and Probability, Holt, Rinehart, and Winston, New York, 1969, pp. 18-86.

8. D. W. Müller, Nonstandard proofs of invariance principles in probability theory, Applications of Model Theory to Algebra, Analysis, and Probability, Holt, Rinehart, and Winston, New York, 1969, pp. 186-194.

9. Salim Rashid, Economies with infinitely many traders, Ph.D. Dissertation, Yale Univ., May, 1976.

10. K. D. Stroyan and W. A. J. Luxemburg, Introduction to the theory of infinitesimals, Academic Press, New York, 1976.

11. V. S. Varadarajan, Measures on topological spaces, Amer. Math Soc. Transl. (2) 48 (1965), 161-228.

Department of Mathematics, Yale University, New Haven, Connecticut 06520

Department of Economics, Dartmouth College, Hanover, New Hampshire 03755 (Current address of Salim Rashid)

Current address (R. M. Anderson): Department of Mathematics, McMaster University, Hamilton, Ontario, Canada L8S 4K1 